Case presentation: The patient was an 83-year-old woman who lived alone. Mild cognitive impairment appeared at the age of 82 years. Soon after, she had the delusional conviction that her family doctor was in love with her. Her symptoms, such as gradually progressive cognitive impairment, cognitive fluctuations, and parkinsonism, indicated DLB. Although small doses of quetiapine, brexpiprazole and risperidone were prescribed for the treatment of the delusion, each of them was discontinued soon because of the adverse reactions. Finally, the delusion was successfully treated with a small dose of blonanserin without sever side effects.

Discussions and Conclusions: This case report suggests the possibility of de Clerambault's syndrome during the early stages of DLB. Recently, psychiatric-onset DLB has increasingly gained attention in recent years. Further accumulation of knowledge about delusions in patients with DLB for an early diagnosis.

\title{
552 - Humanitude Care on Patients with Dementia and Delirium in Acute Hospital Improves Outcomes
}

\author{
Thuy-Anh Giang, Cheng Ling Jie, Chua Min Jia, Philip Yap
}

Background/ Objectives: Older patients with dementia and/or delirium often have challenging behaviours such as refusal of care and aggression. These cause much distress to both healthcare staff and patients, increase burden of care and put older patients at risk of functional decline. Humanitude, a methodology of care developed by Gineste and Marescotti, is a relationship- centred and compassionate care approach that aims to enable patients. The aim of this study is to investigate the effectiveness of Humanitude on older patients' well-being, mobility and activities of daily living (ADLs).

Design: Quasi-experimental, non-equivalent controlled trial design.

Setting: Acute care hospital

Participants: Twenty patients diagnosed with dementia and/ or delirium were recruited from two geriatric wards. Ten were in the Humanitude ward and the other ten in a conventional ward received usual care for older patients served as concurrent controls.

Intervention: Patients in Humanitude ward received Humanitude care by trained healthcare workers during day-to-day care, which is based on the 4 pillars of gaze, speech, touch and verticality (maintaining an upright position). Every patient encounter utilizing Humanitude techniques follow a structured care sequence that helps draw the patient into the care relationship.

Measurements: The outcome measures include Modified Perme ICU Mobility Score, Bradford Wellbeing Profile and Modified Barthel Index (MBI).

Results: There was significant improvement of median score within the Humanitude group from admission to discharge in mobility (admission: 9.0 [0-27] vs discharge 19.5 [1-36], $p=0.002^{* *}$ ), MBI (admission: 20 [0.0-46] vs discharge: 54.3 [3-81], $\mathrm{p}=0.002^{* *}$ ) and well-being (admission: 7.0 [1-15] vs discharge 20.0 [8-26], $\left.p=0.002^{* *}\right)$. The median increase in the score of Humanitude group was also significantly higher than usual care group in mobility (Humanitude: 8 [1-24] vs usual care 0 [-9-16], $p=$ $0.02^{*}$ ), $\mathrm{MBI}$ (Humanitude: 17.5 [3-64] vs usual care $0[-3-17], \mathrm{p}=<0.001^{* * *}$ ), and well-being (Humanitude: 11 [6-20] vs usual care $0[-5-4], p=<0.001 * * *$ ).

Conclusion: Humanitude care improves outcomes in mobility, ADL function and well-being for patients with dementia and/ or delirium in the acute hospital.

(Word count: 329) 